Relevant aspects about design of constructed wetlands coupled to bioelectrochemical systems for wastewater treatment and energy generation

\title{
Aspectos relevantes del diseño de humedales construidos acoplados a sistemas bioelectroquímicos para el tratamiento de efluentes y generación de energía
}

Jorge Antonio Herrera-Cárdenas ${ }^{1}$, Beatriz Lázaro-Rodríguez ${ }^{1}$, Irma Robles-Gutiérrez ${ }^{1}$, Fabricio Espejel-Ayala ${ }^{1}$, María Yolanda Reyes-Vidal ${ }^{1,2 *}$

${ }^{1}$ Centro de Investigación y Desarrollo Tecnológico en Electroquímica, S. C. Parque Tecnológico Querétaro, Sanfandila, Pedro Escobedo, C. P. 76703, Querétaro, México. ${ }^{2}$ Catedrática CONACYT-CIDETEQ, Centro de Investigación y Desarrollo Tecnológico en Electroquímica, S.C. Parque Tecnológico Querétaro, Sanfandila, Pedro Escobedo, C. P. 76703, Querétaro, México.

*Corresponding author.

E-mail address: mreyes@ cideteq.mx (M. Y. Reyes-Vidal).

Article history:

Received: 30 December 2017 / Received in revised form: 27 March 2018 / Accepted: 28 March 2018 / Published online: 1 April 2018.

https://doi.org/10.29267/mxjb.2018.3.2.63

\begin{abstract}
The energy crisis, water scarcity and poor air quality are issues that must be addressed urgently in order to ensure the well-being of the population. The use of alternative technologies such as constructed wetlands (CW) allow the treatment of different wastewaters. When these technologies are coupled to bioelectrochemical systems (BES), the removal of the organic load can be optimized and electrical energy can be generated. Thus, the aim of this work was to perform an analysis of the advances, from 2010 to 2017, on the design of various configurations of $\mathrm{CW}$ systems coupled to BES. It was possible to identify the most important variables that determine the efficiency of removal of pollutants and current generation such as anodic and cathodic materials, area of electrodes, type of macrophytes and material organic removed. Likewise, the values of the organic material removed and the maximum power densities obtained with the three most used macrophytes are presented.
\end{abstract}


Keywords: Constructed wetlands coupled; bioelectrochemical systems; wastewater treatment.

\section{RESUMEN}

La crisis energética, la escasez del agua y la mala calidad del aire son temas que deben ser atendidos urgentemente con el objetivo de garantizar el bienestar de la población. El uso de tecnologías alternativas como los humedales construidos $(\mathrm{HC})$ permiten el tratamiento de aguas residuales de diversa índole; cuando estas tecnologías son acopladas a sistemas bioelectroquímicos (SBE) se optimiza la remoción de la carga orgánica y se puede generar energía eléctrica. Así, el objetivo de este trabajo fue realizar un análisis de los avances, entre los años 2010 a 2017, sobre el diseño de diversas configuraciones de sistemas de HC acoplados a SBE. Se logró identificar las variables más importantes que determinan la eficiencia de remoción de contaminantes y generación de corriente como son los materiales anódico y catódico, el área de los electrodos, el tipo de macrófitas y la carga orgánica. Así mismo, se presentan los valores de la carga orgánica removida y las máximas densidades de potencia obtenidas con las tres macrófitas más utilizadas.

Palabras clave: Humedales construidos acoplados; sistemas bioelectroquímicos; tratamiento de aguas residuales.

\section{INTRODUCCIÓN}

La energía en movimiento está relacionada con el desarrollo de la especie humana, impactando en los aspectos económicos, sociales y de interacción con su medio ambiente. Sin embargo, si el suministro de energía es constante y las principales fuentes de abastecimiento son finitas, la economía como subsistema dependiente de los servicios ecosistémicos no puede crecer infinitamente (Goldin \& Winters, 1995). El uso desmedido de combustibles fósiles incrementa las emisiones de gases de efecto invernadero impactando negativamente sobre la temperatura del planeta (Partdrige et al., 2017). Esta preocupación impulsa el abastecimiento energético a partir de fuentes de energía alternativas, las cuales se obtienen de fuentes naturales inagotables debido a su capacidad de regenerarse naturalmente (Guzmán et al., 2017; Esperbent, 2015). Estas representan un potencial inmenso para mitigar los efectos dramáticos del consumo energético en continuo crecimiento (Coviello, 2003).

Los sistemas biolectroquímicos (SBE) son el resultado de los avances en materia del manejo integral de los residuos, que a través de técnicas biotecnológicas buscan la valorización de los desechos en sus diversas formas (líquida o sólida). Las celdas de combustible microbianas (CCM, del termino en inglés "Microbial fuel cell") son un tipo de SBE que utilizan bacterias, que crecen sobre un soporte sólido, como biocatalizador para oxidar la materia orgánica y producir bioenergía (Wu et al., 2016). La materia orgánica sirve como sustrato para el metabolismo de bacterias electrogénicas que generaran electrones transferidos al ánodo, los cuales viajan al cátodo mediante un circuito externo (Gao et al., 2017).

Existe amplio interés por el estudio de las CCM y más recientemente hacia diversas configuraciones de los SBE, como fuentes potenciales para la generación de energía 
renovable; es un campo en constante expansión que combina la actividad biológica con reacciones electroquímicas abióticas (Logan \& Rabaey, 2012; Logan \& Elimelech, 2012). De acuerdo al número de publicaciones sobre CCM incluidas en ScienceDirect, del 2010 a la fecha se reportan 17,940 trabajos, de los cuales 10,106 son investigaciones originales y 2,865 corresponde a artículos de revisión. Se tiene especial atención a las celdas de combustible microbianas debido a su fácil operación y capacidad para utilizar residuos de diversa índole como sustratos (Rahimnejad et al., 2015), convirtiendo la energía química en electricidad mediante procesos microbianos (Bentley, 2002).

Los humedales artificiales o construidos ( $\mathrm{HC}$, del término en inglés "Constructed Wetlands") son sistemas de depuración de aguas residuales domésticas, o industriales, que imitan los fenómenos que se desarrollan en sistemas naturales. En estos sistemas ocurren diversos procesos físicos (filtración, adsorción, sedimentación, volatilización), químicos (foto degradación, reacciones de óxido reducción) y biológicos (Faulwetter et al., 2009) favoreciendo la eliminación de diversos contaminantes. Estos sistemas reciben amplia atención para el tratamiento de aguas residuales domésticas en pequeñas comunidades debido a sus ventajas de fácil mantenimiento y alta capacidad de autopurificación (Onn et al., 2015).

Tanto las CCM como los HC poseen zonas aerobias y anaerobias, donde se producen simultáneamente reacciones de óxido - reducción (Liu et al., 2014). Los sistemas de HC acoplados a CCM (HC-CCM) son una tecnología innovadora que integra ambos dispositivos para el tratamiento de aguas residuales y generación de energía, confiables y adecuados para la operación a largo plazo (Villaseñor et al., 2013; Corbella et al., 2014). La eficiencia de remoción de diversos contaminantes por este sistema integrado se ha reportado para fármacos (Zhang et al., 2017), residuos de la industria ganadera (Doherty et al., 2015b; Zhao et al., 2013), colorantes azoicos (Yadab et al., 2012; Fang et al., 2015; Fang et al., 2016) y subproductos del tratamiento de aguas residuales (Corbella et al., 2016; Liu et al., 2017; Wu et al., 2017).

Los avances publicados acerca de HC-CCM a nivel global presentan mínimas contribuciones de parte de los países en vías de desarrollo, siendo una alternativa tecnológica aún en investigación y con pocos resultados de aplicación a gran escala. Por lo tanto, el objetivo de este trabajo fue realizar un análisis de los trabajos publicados sobre humedales construidos acoplados a celdas de combustible microbianas, con la finalidad de identificar las variables críticas para el diseño de tales sistemas. Se realizó una revisión bibliográfica en los 50 artículos más recientes, obtenidos en las bases de datos de Sciencedirect, SCIELO y EBSCO, mediante las palabras clave: "microbial" and "fuel" and "cell" and "constructed" and "wetlands" and "coupled", en el periodo comprendido entre 2010 a 2017, haciendo énfasis en parámetros como: sustrato, material y tamaño de los electrodos, temperatura de operación, tipo de macrófita, medio poroso y biopelículas. Los datos se recolectaron utilizando un formulario que contiene información relacionada con tales parámetros, para homogenizar y analizar los datos obtenidos, identificando las características clave de estos sistemas. 


\section{COMPONENTES DE UN SISTEMA ACOPLADO HUMEDAL CONSTRUIDO- CELDA DE COMBUSTIBLE MICROBIANA (HC-CCM)}

Los sistemas más comunes de HC-CCM están integrados por la cámara anaeróbica (ánodo), cámara aeróbica (cátodo), comunidad bacteriana, sistema de alimentación y drenado, soporte poroso, resistencia, material vegetal (Fang et al., 2017) y un separador conectando las dos cámaras que debe garantizar el intercambio de protones (Rho \& Woo, 2017) (Fig. $1)$.

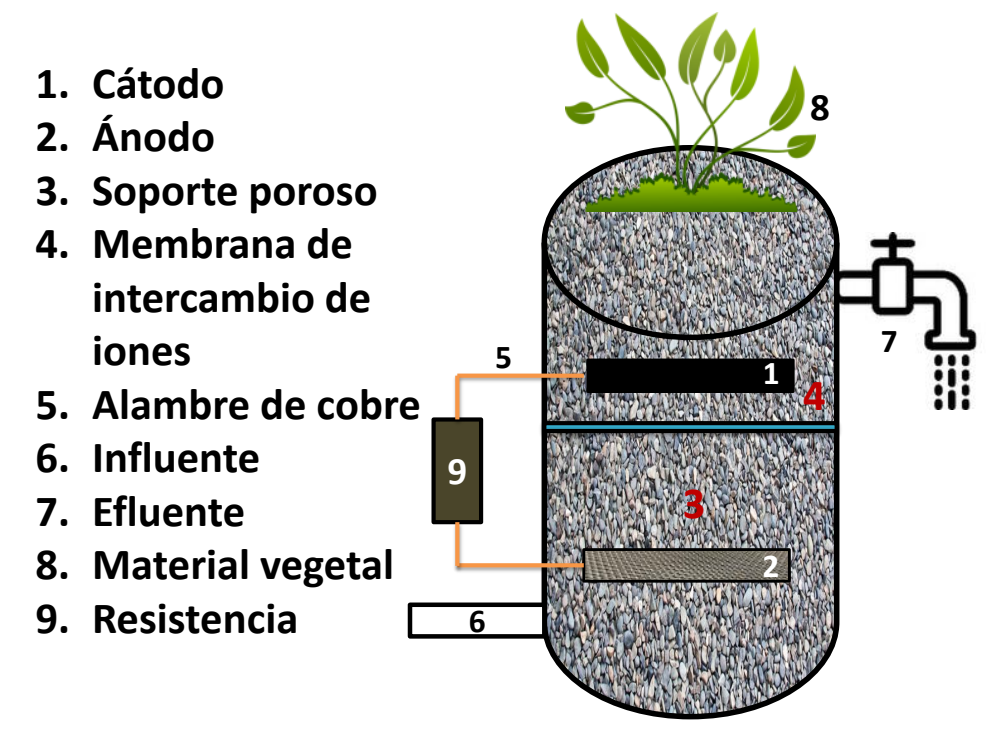

Fig. 1. Componentes básicos de un sistema de HC-CCM.

Los electrodos incluyen ánodo y cátodo, en el ánodo las bacterias oxidan la materia orgánica produciendo electrones que son transferidos mediante un circuito externo hacia el cátodo, la producción y el consumo de electrones tiene como resultado una diferencia de potencial entre ambos electrodos (Timmer et al., 2010). Para lograr una mejor producción de electricidad y favorecer la fijación de película microbiana, el material del electrodo debe tener propiedades como biocompatibilidad, conductividad electrónica, estabilidad química, área de superficie volumétrica, alta porosidad superficial y bajo costo (Saratale et al., 2017).

\subsection{Material de los electrodos}

Cualquier configuración de un sistema bioelectroquímico acoplado a un humedal construido posee dos electrodos (ánodo y cátodo) que pueden fabricarse de diferentes materiales. Los materiales más reportados son grafito, carbón activado granular, fieltro de fibra de carbono y malla de acero inoxidable. Los autores señalan que para reducir el costo de estos sistemas es necesario utilizar materiales baratos, tales como los ánodos de fieltro de carbón, tela de carbono y carbón activado (Gau et al., 2017). Los electrodos de fieltro de grafito generan tres veces más electricidad en comparación con la varilla de grafito (Wen $e t$ al., 2012).

Tsai et al. (2009) reportaron el uso de nanotubos de carbono para mejorar la generación de densidad de potencia en las CCM, sin embargo, observaron una inhibición del crecimiento 
bacteriano que provoca la muerte celular. El material de los electrodos tiene un efecto significativo sobre el rendimiento de estos sistemas, debido a la propiedad de intercambio de electrones con la biopelícula electroquímica activa. Existen muchos materiales que presentan diferencia en la porosidad, área de superficie y conductividad que afectan directamente a la adhesión de los microorganismos (Saratale et al., 2017).

\subsection{Tamaño de los electrodos}

El área de los electrodos proporciona un área más grande para el establecimiento de bacterias, además de mejorar el intercambio de electrones entre el electrodo y la bacteria, debido a una mayor superficie para el establecimiento de las comunidades bacterianas, por lo que el área tiene un efecto directo sobre el rendimiento de la densidad de corriente (Chen et al., 2011). Wang et al. (2016) la superficie del electrodo promueve el crecimiento de bacterias electroactivas que son capaces de utilizar materia orgánica para generar electrones. No obstante, Song et al. (2017) reportaron que, en comparación con el ánodo, el cátodo puede desempeñar un papel más destacado en el rendimiento energético del reactor. Fang et al. (2017) reportaron que el aumento del área del cátodo promueve los procesos de degradación de colorantes y el rendimiento de generación de electricidad, pero que existen limitantes ya que cátodos de diámetros demasiado grandes pueden ser desfavorables para el rendimiento de los sistemas HC-CCM.

\subsection{Sustrato}

La carga orgánica afecta en gran medida el rendimiento del sistema, sin embargo, es necesario lograr un equilibro entre proporcionar los elementos orgánicos suficientes para la oxidación en el ánodo y limitar la cantidad que llega al cátodo (Doherty et al., 2015). Liu et al. (2014) observaron que una concentración de DQO superior a $250 \mathrm{mg} / \mathrm{L}$ disminuye la densidad de potencia obtenida, debido a que se incrementa la posibilidad de que una alta concentración de materia orgánica llegue al cátodo, provocando un aumento en la demanda de oxígeno, con lo que se limita el oxígeno disponible para las reacciones de reducción necesarias para cerrar el circuito. Por otra parte, Fang et al. (2015) reportaron que a una concentración de $300 \mathrm{mg} / \mathrm{L}$ de DQO se obtuvo un mayor rendimiento del HC-CCM, logrando una tasa de decoloración y densidad de potencia de $95.6 \%$ y $0.852 \mathrm{~W} / \mathrm{m}^{3}$, respectivamente.

Los rendimientos electroquímicos de los BES y las propiedades biológicas de la biopelícula se relacionan con el tipo de sustrato (Catal et al., 2008). La carga de sustrato influye significativamente en las características y el crecimiento de la biopelícula, por lo que la velocidad de carga es esencial para mejorar la eficiencia del proceso (Mohan et al., 2014). Debido a que estos sistemas han comenzado a desarrollarse recientemente, se reportan efluentes sintéticos que permiten el suministro controlado de nutrientes y contaminantes de interés. Es importante destacar, que se requiere de una fuente de carbono como es glucosa, acetato o ácidos grasos. Así como un aporte de macronutrientes proveniente de las sales que se mezclan en concentraciones establecidas, principalmente, cloruro de amonio, fosfato monobásico y dibásico de sodio, cloruro de potasio y bicarbonato de sodio (Fang et al., 2016), y de una solución de micronutrientes que suministre elementos como $\mathrm{Zn}, \mathrm{Cd}, \mathrm{Mg}$, Mn, Ca y Mo (Yadav et al., 2012). Según el análisis de los trabajos publicados, en los 
sistemas acoplados HC-CCM ciertos contaminantes son usados como sustrato del metabolismo bacteriano, entre ellos se tienen a los colorantes azoicos y algunos contaminantes que actúan como nutrientes, por ejemplo, amonio, nitratos y fosfatos (Zhi et al., 2015; Gao et al., 2017; Liu et al., 2018).

\subsection{Medio poroso}

El medio poroso representa un soporte para el material vegetal, además de amortiguar el flujo del agua a través del sistema, lo cual favorece los procesos de sedimentación, filtración y adsorción, así como de la formación de biopelículas; en el caso de las CCM funciona como un soporte que estabiliza la posición de los electrodos. Los materiales reportados para estos sistemas son: uno que incluye solamente grava (Onn et al., 2017) y otro que utiliza lodo de alumbre o de cuarzo (Wu et al., 2017; Xu et al., 2016; Wang et al., 2016a). La función que cumple este componente depende de las características del medio poroso ya que corresponde con el área superficial obtenida, lo que juega un papel importante en el establecimiento de las comunidades bacterianas y su relación con la optimización de procesos de remoción.

\subsection{Biopelículas}

A lo largo del microcosmos HC-CCM existe una abundancia de superficies alternativas, además del electrodo, para la adhesión y el crecimiento bacterianos (biopeliculas). Estas bacterias pueden oxidar las sustancias orgánicas en las aguas residuales, liberando electrones que no pueden ser capturados por el ánodo (Doherty et al., 2015a). Una biopelícula se conoce como una reunión de microrganismos (bacterias, algas, hongos) con células que se adhieren mutuamente y a una superficie. A menudo, las células están integradas dentro de una matriz polimérica autoproducida (Saratale et al., 2017). La formación de estas biopelículas es un proceso continuo y secuencial que incluye transporte, fijación, formación de microcolonias y maduración de biopelículas en la superficie del electrodo (Sauer et al., 2002; Mohan et al., 2014). Es un proceso muy complejo, afectado directamente por el tipo de sustrato y su concentración, condiciones de operación, materiales de electrodo, tipo de cepa de los microorganismos que incluye cultivo puro o mixto, y sus rutas metabólicas (Saratale et al., 2017).

La diversidad bacteriana depende del origen del inóculo y guarda relación directa con el rendimiento energético; a la fecha se han reportado más de 20 bacterias electrogénicas diferentes, incluyendo especies de los géneros: Desulfuromonas, Pseudomonas, Desulfobulbus, Thermincola, Geothrix, Ochrobactrum, Acidiphilium, Rhodoferax, Comamonas, Shewanella, Klebsiella, Enterobacter, Rhodopseudomonas, Citrobacter, Geobacter, Geopsychrobacter, Arcobacter, Aeromonas, Propionibacterium, Clostridium, entre otras (Corbella et al., 2015). Clostridium butyricum fue el primer microorganismo electrogénico Gram positivo (Beckers et al., 2013), un tipo de bacteria anaeróbica estricta, que puede producir electricidad metabolizando almidón, sacarosa y otros polisacáridos. Clostridium beijerinckii también puede producir electricidad usando almidón, melaza, glucosa y ácido láctico (Zhang et al., 2013). Paracoccus denitrificans es una bacteria desnitrificante común en tratamiento de aguas residuales, y considerada en diversos estudios como microorganismo electrogénico (He et al., 2014). Una delta-proteobacteria ampliamente conocida Geobacter sulfurreducens es considerada uno de los 
microorganismos electrogénicos más comunes. En condiciones anaeróbicas, estas bacterias pueden oxidar directamente el ácido acético y transferir electrones al electrodo ( Lu et al., 2015).

\subsection{Tipo de macrófita}

El rendimiento de los HC-CCM es obstaculizado por la resistencia interna (Doherty et al., 2015). Los cátodos en la interface aire-agua se utilizan para incrementar la eficiencia de las reacciones catódicas, sin embargo, su uso puede exacerbar el problema de resistencia interna, aumentando la distancia entre ambos electrodos. Esta distancia se puede reducir, utilizando el oxígeno excretado por las plantas en estos sistemas (Chen et al., 2012).

Las macrófitas desempeñan un papel fundamental en la configuración de las HC-CCM, ya que estas pueden promover el rendimiento del cátodo debido a la inyección de oxígeno a través de la zona radicular, jugando un papel positivo para el potencial del cátodo debido a que pueden mejorar el rendimiento del reactor en la generación de electricidad (Yang et al., 2016), incrementar la cantidad de microorganismos (Fang et al., 2013), cambiar las propiedades de la superficie y reducir la resistencia interna del cátodo. Según Fang et al. (2017) la presencia de plantas en el cátodo aumentó el voltaje; sin embargo, no aumentaron la densidad de corriente de intercambio del cátodo. Fang et al. (2013) ya había reportado que la presencia de plantas afectó significativamente la densidad de potencia, obteniendo máxima densidades de potencia de $0.302 \mathrm{~W} / \mathrm{m}^{3}$ en un sistema plantado y de $0.191 \mathrm{~W} / \mathrm{m}^{3}$ en uno no plantado, atribuyendo a que la presencia de plantas en el cátodo incrementó la cantidad de microorganismos.

En la Tabla 1 se presenta el rendimiento de diversos sistemas HC-CCM con respecto a algunos criterios de diseño. Se observa que los valores de máxima densidad de potencia (MDP) normalizados con respecto al área del ánodo y relacionados con el rendimiento de los sistemas no depende únicamente del área del electrodo y la concentración de sustrato, sino también del material del electrodo, mostrando un mejor rendimiento los de malla de acero inoxidable con carbón activado granular en comparación con los de fieltro de fibra de carbono. Cuando el rendimiento del sistema se normaliza al volumen del electrodo, ocurre algo similar, con un volumen de $1.5 \mathrm{~L}$ y una concentración de sustrato de $500 \mathrm{mg} / \mathrm{L}$ (DQO) se logra un rendimiento menor en comparación con un sistema de $1.1 \mathrm{~L}$ y una concentración de sustrato de $583 \mathrm{mg} / \mathrm{L}$ (DQO), mostrando mejores resultados los sistemas con electrodos elaborados con barras de grafito, en comparación con los de malla de acero inoxidable y grafito granular. Por lo que se podría sugerir que la eficiencia no solo depende del área del electrodo y la concentración de sustrato, sino también del material de electrodo. Entre los materiales más eficientes para la fabricación del ánodo están el grafito y la malla de acero inoxidable con carbón activado granular, sin embargo, si se tiene el objetivo de escalar estos sistemas el fieltro de fibra de carbono es uno de los materiales más accesibles, como ya se mencionó anteriormente. 
Tabla 1. Resumen de las máximas densidades de potencia obtenidas respecto al área del ánodo y concentración de sustrato y tipo de macrófita.

\begin{tabular}{|c|c|c|c|c|c|c|}
\hline Macrófita & MDP & $\begin{array}{l}\text { Tipo de } \\
\text { efluente }\end{array}$ & Ánodo & $\begin{array}{l}\text { Área / } \\
\text { Volumen }\end{array}$ & $\begin{array}{l}\text { Concentración } \\
\text { de sustrato } \\
\mathrm{mg} / \mathrm{L}\end{array}$ & Referencia \\
\hline $\begin{array}{l}\text { Canna } \\
\text { indica }\end{array}$ & $18 \mathrm{~mW} / \mathrm{m}^{2}$ & Sedimento & DG & $122 \mathrm{~cm}^{2}$ & na & $\begin{array}{l}\text { Lu et al., } \\
2015\end{array}$ \\
\hline $\begin{array}{l}\text { Canna } \\
\text { indica }\end{array}$ & $8.91 \mathrm{~mW} / \mathrm{m}^{2}$ & $\begin{array}{c}\text { Agua } \\
\text { sintética }\end{array}$ & FFC & $154 \mathrm{~cm}^{2}$ & 120 (glucosa) & $\begin{array}{l}\text { Wang et } \\
\text { al., } 2017\end{array}$ \\
\hline $\begin{array}{l}\text { Canna } \\
\text { indica }\end{array}$ & $3.02 \mathrm{~mW} / \mathrm{m}^{2}$ & $\begin{array}{c}\text { Agua } \\
\text { sintética }\end{array}$ & FFC & $154 \mathrm{~cm}^{2}$ & $120($ glucosa $)$ & $\begin{array}{l}\text { Wang et } \\
\text { al., 2016a }\end{array}$ \\
\hline $\begin{array}{l}\text { Canna } \\
\text { indica }\end{array}$ & $\begin{array}{l}10.77 \\
\mathrm{~mW} / \mathrm{m}^{2}\end{array}$ & $\begin{array}{l}\text { Agua } \\
\text { sintética }\end{array}$ & CAG/MAI & $493 \mathrm{~cm}^{2}$ & 197 (acetato) & $\begin{array}{l}\text { Xu et al., } \\
\quad 2017\end{array}$ \\
\hline $\begin{array}{l}\text { Phragmite } \\
\text { s australis }\end{array}$ & $4.4 \mu \mathrm{W} / \mathrm{m}^{2}$ & $\begin{array}{l}\text { Residuos } \\
\text { ganaderos }\end{array}$ & PG & $71 \mathrm{~cm}^{2}$ & $\mathrm{np}$ & $\begin{array}{l}\text { Zhao et al., } \\
\quad 2013\end{array}$ \\
\hline $\begin{array}{l}\text { Typha } \\
\text { latifolia } L\end{array}$ & $\begin{array}{l}87.79 \mathrm{~mW} / \\
\mathrm{m}^{2}\end{array}$ & $\begin{array}{c}\text { Agua } \\
\text { sintética }\end{array}$ & MAI/CAP & $70 \mathrm{~cm}^{2}$ & 500 (DQO) & $\begin{array}{l}\text { Xu et al., } \\
2016\end{array}$ \\
\hline $\begin{array}{l}\text { Typha } \\
\text { latifolia L }\end{array}$ & $\begin{array}{l}36.48 \\
\mathrm{~mW} / \mathrm{m}^{3}\end{array}$ & $\begin{array}{c}\text { Agua } \\
\text { sintética }\end{array}$ & $\mathrm{CA}$ & $\begin{array}{l}2544.7 \\
\mathrm{~cm} 3\end{array}$ & 107.1 (acetato) & $\begin{array}{l}\text { Onn et al., } \\
2017\end{array}$ \\
\hline $\begin{array}{l}\text { Canna } \\
\text { indica }\end{array}$ & $\begin{array}{l}42.23 \mathrm{~mW} / \\
\mathrm{m}^{3}\end{array}$ & $\begin{array}{c}\text { Agua } \\
\text { sintética }\end{array}$ & GGR & $207.8 \mathrm{~cm}^{3}$ & 500 (glucosa) & $\begin{array}{l}\text { Srivastava } \\
\text { et al., } 2015\end{array}$ \\
\hline $\begin{array}{l}\text { Phragmite } \\
\text { s australis }\end{array}$ & $0.268 \mathrm{~W} / \mathrm{m}^{3}$ & $\begin{array}{l}\text { Residuos } \\
\text { ganaderos }\end{array}$ & BG & $1100 \mathrm{~cm}^{3}$ & 583 (DQO) & $\begin{array}{l}\text { Doherty et } \\
\text { al., 2015a }\end{array}$ \\
\hline $\begin{array}{l}\text { Phragmite } \\
\text { s australis }\end{array}$ & $0.276 \mathrm{~W} / \mathrm{m}^{3}$ & $\begin{array}{l}\text { Residuos } \\
\text { ganaderos }\end{array}$ & BG & $1100 \mathrm{~cm}^{3}$ & 583 (DQO) & $\begin{array}{l}\text { Doherty et } \\
\text { al., } 2015\end{array}$ \\
\hline $\begin{array}{l}\text { Phragmite } \\
\text { s australis }\end{array}$ & $0.17 \mathrm{~W} / \mathrm{m}^{3}$ & $\begin{array}{l}\text { Agua } \\
\text { sintética }\end{array}$ & CAG & $2100 \mathrm{~cm}^{3}$ & 200 (Glucosa) & $\begin{array}{l}\text { Song et al., } \\
2017\end{array}$ \\
\hline $\begin{array}{l}\text { Phragmite } \\
\text { s australis }\end{array}$ & $\begin{array}{l}1413.25 \\
\mathrm{~mW} / \mathrm{m}^{3}\end{array}$ & $\begin{array}{l}\text { Agua } \\
\text { sintética }\end{array}$ & MAI/GG & $1500 \mathrm{~cm}^{3}$ & 500 (DQO) & $\begin{array}{c}\text { Xu et al., } \\
2017 \mathrm{a}\end{array}$ \\
\hline
\end{tabular}

DG: disco de grafito; FFC: fieltro de fibra de carbono; CAG: carbón activado granular; PG: placas de grafito; MAI: malla de acero inoxidable; GA: carbón activado; $\boldsymbol{C A P :}$ carbón activado pulverizado; GG: grafito granular; BG: barras de grafito; GGR: grafito granular; GG: grava de grafito; np: no reportado; na: no aplica. 


\section{OPERACIÓN DE LOS SISTEMAS HC-CCM}

\subsection{Temperatura de operación}

La temperatura es un factor importante en los procesos biotecnológicos microbianos (Liu et al., 2005). Patil et al. (2010) demostraron que la temperatura influye en la formación de biopelículas y en el rendimiento electrocatalítico. El rango óptimo de temperatura operativa es de 25 a $35^{\circ} \mathrm{C}$, aunque los procesos de remoción se desarrollan mejor a $35^{\circ} \mathrm{C}$ (Tee et al., 2017), debido a que los descensos de temperatura afectan la diversidad de las comunidades bacterianas.

Fang et al. (2013) reportaron que con un sistema cuyo volumen anódico de aproximadamente $7 \mathrm{~L}$ y una temperatura operativa de $25 \pm 2^{\circ} \mathrm{C}$ se obtuvo una densidad máxima de potencia (DMP) de $0.302 \mathrm{~W} / \mathrm{m}^{3}$. Por otra parte, Lu et al. (2015) reportaron una MDP de $18 \mathrm{~mW} / \mathrm{m}^{2}$ con un sistema operado a $23 \pm 2^{\circ} \mathrm{C}$ y un ánodo de $20.3 \mathrm{~cm}^{2}$ de tela de carbono. Wang et al. (2017) lograron una MDP de $21.53 \mathrm{~mW} / \mathrm{m}^{2}$ con una temperatura de operación de 17 a $38^{\circ} \mathrm{C}$ y un ánodo de fieltro de fibra de carbono de $10 \mathrm{~cm}^{2}$. Finalmente, Song et al. (2017) reportaron una MDP de $0.17 \mathrm{~W} / \mathrm{m}^{3}$ con un sistema cuyo ánodo fue de carbón activado granular, un volumen de $2.1 \mathrm{~L}$ y temperatura de operación del sistema de $28 \pm 2^{\circ} \mathrm{C}$.

\subsection{Evaluación del rendimiento}

El rendimiento de estos sistemas se evalúa de acuerdo a variables como resistencia interna, diferencia de potencial, máxima densidad de potencia (MDP) y máxima densidad de corriente (MDC). La eficiencia de biodegradación (eliminación de compuestos orgánicos) se relaciona con los cambios en la demanda química de oxígeno (DQO), expresada como porcentaje de remoción. Los cambios en estas variables se determinan mediante lecturas de potencial en distintos periodos de tiempo y mediciones de DQO en el influente y efluente del sistema. Se ha reportado que la densidad de corriente y la densidad de potencia disminuyen a medida que se incrementa la concentración del contaminante (Fang et al., 2015), no obstante, otros estudios han reportado que la producción de bioenergía se incrementa en función de la concentración de sustrato (Wang et al., 2016).

Las mediciones de voltaje $(\mathrm{U})$ permiten determinar parámetros como la corriente $(\mathrm{I}=\mathrm{V} / \mathrm{R})$ y la potencia $(\mathrm{P}=\mathrm{V} * \mathrm{I})$. Cuando estos valores se dividen por el área de las superficies $\left(\mathrm{m}^{2}\right)$ o el volumen de la cámara anódica $\left(\mathrm{m}^{3}\right)$ se obtiene la densidad de corriente $(\mathrm{J})$ y de potencia $(\mathrm{P})$ (Zhao et al., 2013; Doherty et al., 2015), que al graficar con respecto al tiempo se observa el máximo valor de ambos parámetros. De igual manera, estos cambios pueden analizarse mediante curvas de polarización (Logan et al., 2006), relacionando P contra J y U. Este tipo de gráficas proporcionan información determinante para analizar el rendimiento de la celda (Appleby, 1988), ofreciendo información de la activación causada por fenómenos como la lenta cinética del electrodo, la resistencia de flujo de iones en el electrolito y la resistencia al flujo de electrones a través de los electrodos, así como, pérdidas relacionadas con el transporte de masa (Xu et al., 2017). Las curvas de polarización se obtienen variando las resistencias durante ciertos periodos de tiempo (Wang et al., 2017), reportándose rangos de variación desde 50 hasta 80,000 $\Omega$ (Wang et al., 2017a), de 20 a 580,000 $\Omega$ (Xu et al., 2017), 1 a $680,000 \Omega$ (Türker \& Yakar, 2017) y de 5 a 100,000 $\Omega$ (Fang et al., 2016). 
La eficiencia coulómbica (EC, del término en inglés "Coulombic Efficiency") es otro de los parámetros que evalúa el rendimiento del sistema (Ecuación 1), permitiendo obtener la fracción de energía que se puede generar a partir de un sustrato determinado. Este parámetro combina la cantidad de electrones cosechados como equivalentes actuales y totales contenidos en la materia orgánica disponible para el metabolismo oxidativo microbiano (DQO) (Logan, 2009).

$E C=\frac{I}{F x \frac{4}{32} x Q_{\text {in }} x \Delta D Q O} \quad$ Ecuación 1

En la Ecuación 1 es necesario considerar a I como la corriente, F es la constante de Faraday $\left(96485 \mathrm{C} / \mathrm{mol} \mathrm{e}^{-}\right), 32$ que corresponde con la masa molar del $\mathrm{O}_{2}$, 4 es el número de electrones obtenidos por la reacción de reducción de 1 molar de $\mathrm{O}_{2}$, Qin es el caudal de afluencia volumétrica del HC-CCM (L/s) y $\triangle \mathrm{DQO}$ es la diferencia en la DQO entre el afluente y efluente del ánodo.

De acuerdo a Srivastava et al. (2017) teniendo las mediciones de EC se puede estimar el balance de carbono mediante las Ecuaciones 2 y 3.

$Y=\frac{X}{\Delta D Q O} \quad$ Ecuación 2

$\varphi=1-E C-Y \quad$ Ecuación 3

Considerando que, $\mathrm{Y}$ es el rendimiento celular, $\mathrm{X}$ es la biomasa (expresada en g de COD) producida a través del tiempo, $\varphi$ es el balance de DQO y EC es la eficiencia coulómbica (Srivastava et al., 2017).

En la Tabla 1 se muestran los resultados más relevantes publicados para la máxima densidad de corriente, los materiales más utilizados en la fabricación de los electrodos, así como las especies de macrófitas y el tipo de efluente. Adicionalmente se realizó un análisis de la correlación entre el volumen del sistema y la densidad de potencia obtenida, sin encontrar relaciones entre ambas variables, por lo que es conveniente realizar un análisis detallado mediante un diseño experimental elaborado a partir de la información obtenida del efecto los factores y sus posibles interacciones, durante el escalamiento de estos sistemas.

El reporte de estas variables permite el análisis comparativo entre los sistemas, sin embargo, es necesario que haya un consenso entre los grupos de investigación a nivel mundial para unificar las mediciones y la forma de reportarlas para llevar a cabo tal tarea, ya que en algunas ocasiones se presentan solo los valores de uno de los parámetros o es 
nula la información para realizar los cálculos del resto. Además, que existen estudios que señalan otros factores, poco reportados, que son determinantes para el rendimiento de estos sistemas (HC-CCM) como el $\mathrm{pH}$, los donantes y aceptores de electrones, la matriz (soporte), espacio entre electrodos, factores de influencia (ambientales), parámetros de operación y la presencia de plantas (Li et al., 2016).

\section{ANÁLISIS Y PERSPECTIVAS}

De los trabajos revisados, más del $90 \%$ se realizan a escala laboratorio, enfocados en dos temas principales de las variables de entrada, como son: a) el efecto de diversas características de los electrodos (material, posición, área superficial y distancia entre ellos) y b) el efecto de las macrófitas y del tipo de efluente sobre el desarrollo de las comunidades bacterianas. De estas variables, el incremento en la superficie disponible aumenta el rendimiento del ánodo porque permite la adhesión de los microorganismos (Roh \& Woo, 2017) así como el tipo y concentración de sustrato, cepa (cultivo puro o mixto), material del electrodo y condiciones de operación influyen en la formación de la biopelícula (Saratale et al., 2017). Según Nam et al. (2010) las variables críticas de diseño de los sistemas HCCCM son el material y la distancia entre los electrodos, el tiempo de residencia hidráulica y la carga orgánica o concentración de sustrato.

Aunque está determinada la eficiencia de estos sistemas en la depuración de aguas residuales y generación de energía es necesario evaluarlos a una escala de mesocosmos, con el objetivo de considerar el efecto de diversos factores ambientales sobre su eficiencia operativa y hacer inferencias más precisas respecto a su funcionamiento. Los humedales construidos constituyen una tecnología económicamente viable para la depuración de agua en comunidades rurales o con problemas de escasez de agua. Estos sistemas son eficientes en la remoción de una amplia gama de contaminantes y efluentes de diversa naturaleza. La limitante que presentan es el área superficial que se necesita para su instalación, sin embargo, el acoplamiento a CCM, permitiría una significativa reducción del área superficial mediante el uso de microorganismos electroactivos y la aplicación de pulsos eléctricos, incrementando de igual manera la eficiencia de remoción (Salas et al., 2017).

Es necesario que el estudio de los SBE acoplados a $\mathrm{HC}$ se realice de manera estructurada mediante el uso de diversas herramientas estadísticas que permitan identificar las variables criticas de diseño y evaluar el efecto de tales variables así como las interacciones entre ellas, de tal manera que se puedan obtener los criterios básicos de diseño para el escalamiento de estos sistemas; sobre todo ante la necesidad de fuentes de energía alternativas y vertido de efluentes urbanos que cumplan con los estándares de calidad establecidos en la legislación Nacional.

\section{AGRADECIMIENTOS}

Herrera-Cárdenas agradece al Fondo Sectorial CONACYT-Secretaria de Energía Sustentabilidad Energética por la beca de posdoctorado otorgada. Al proyecto 246052 del Fondo CONACYT-SENER-Sustentabilidad Energética y al proyecto 258159 del Fondo SEP-CONACYT-Ciencia Básica. 


\section{CONFLICTO DE INTERESES}

Los autores declaran que no existe conflicto de intereses.

\section{REFERENCIAS}

Appleby, A. J., \& Foulkes, F.R. 1988. Fuel Cell Handbook, New York: Van Nostrand Reinhold, Co. Inc.

Beckers, L., Hiligsmann, S., Lambert, S. D., Heinrichs, B., \& Thonart, P. 2013. Improving effect of metal and oxide nanoparticles encapsulated in porous silica on fermentative biohydrogen production by Clostridium butyricum. Bioresource Technology. 133: 109-117.

Bentley, R. W. 2002. Global oil \& gas depletion: an overview. Energy Policy. 30(3): 189205.

Camacho, J. V., Romero, L. R., Marchante, C. F., Morales, F. F., \& Rodrigo, M. R. 2017. The salinity effects on the performance of a constructed wetland-microbial fuel cell. Ecological Engineering. 107: 1-7.

Castro-Martínez, E., Olmos-Peñuela, J., \& Fernández-de-Lucio, I. 2016. La vinculación ciencia-sociedad: estereotipos y nuevos enfoques. Journal of Technology Management \& Innovation. 11 (2): 121-129.

Catal, T., Li, K., Bermek, H., \& Liu, H. 2008. Electricity production from twelve monosaccharides using microbial fuel cells. Journal of Power Sources. 175(1): 196-200.

Chen, S., Hou, H., Harnisch, F., Patil, S. A., Carmona-Martinez, A. A., Agarwal, S., \& Schröder, U. 2011. Electrospun and solution blown three-dimensional carbon fiber nonwovens for application as electrodes in microbial fuel cells. Energy \& Environmental Science. 4(4): 1417-1421.

Chen, Z., Huang, Y. C., Liang, J. H., Zhao, F., \& Zhu, Y. G. 2012. A novel sediment microbial fuel cell with a biocathode in the rice rhizosphere. Bioresource Technology. 108: 55-59.

Corbella, C., García, J., \& Puigagut, J. 2016. Microbial fuel cells for clogging assessment in constructed wetlands. Science of the Total Environment, 569, 1060-1063.

Corbella, C., Garfí, M., \& Puigagut, J. 2014. Vertical redox profiles in treatment wetlands as function of hydraulic regime and macrophytes presence: surveying the optimal scenario for microbial fuel cell implementation. Science of the Total Environment. 470: 754-758.

Coviello, M. 2003. Entorno internacional y oportunidades para el desarrollo de las fuentes renovables de energía en los países de América Latina y el Caribe. CEPAL.

de la Varga, D., Soto, M., Arias, C. A., van Oirschot, D., Kilian, R., Pascual, A., \& Álvarez, J. A. 2017. Constructed wetlands for industrial wastewater treatment and removal 
of nutrients. In Technologies for the Treatment and Recovery of Nutrients from Industrial Wastewater (pp. 202-230). IGI Global.

Doherty, L., Zhao, X., Zhao, Y., \& Wang, W. 2015. The effects of electrode spacing and flow direction on the performance of microbial fuel cell-constructed wetland. Ecological Engineering. 79: 8-14.

Doherty, L., Zhao, Y., Zhao, X., \& Wang, W. 2015b. Nutrient and organics removal from swine slurry with simultaneous electricity generation in an alum sludge-based constructed wetland incorporating microbial fuel cell technology. Chemical Engineering Journal. 266: 74-81.

Doherty, L., Zhao, Y., Zhao, X., Hu, Y., Hao, X., Xu, L., \& Liu, R. 2015a. A review of a recently emerged technology: Constructed wetland-Microbial fuel cells. Water Research. 85: $38-45$.

Esperbent, C. 2015. Motor para el desarrollo de las comunidades: La energía sostenible tiene un importante efecto en la productividad, la salud y la seguridad alimentaria e hídrica de las personas. En América Latina se impulsan proyectos para que las comunidades rurales puedan acceder a fuentes alternativas. RIA. Revista de Investigaciones Agropecuarias. 41(1): 21-27.

Fang, Z., Cao, X., Li, X., Wang, H., \& Li, X. 2017. Electrode and azo dye decolorization performance in microbial-fuel-cell-coupled constructed wetlands with different electrode size during long-term wastewater treatment. Bioresource Technology. 238: 450-460.

Fang, Z., Cheng, S., Wang, H., Cao, X., \& Li, X. 2017. Feasibility study of simultaneous azo dye decolorization and bioelectricity generation by microbial fuel cell-coupled constructed wetland: substrate effects. RSC Advances. 7(27): 16542-16552.

Fang, Z., Song, H. L., Cang, N., \& Li, X. N. 2015. Electricity production from Azo dye wastewater using a microbial fuel cell coupled constructed wetland operating under different operating conditions. Biosensors and Bioelectronics. 68: 135-141.

Fang, Z., Song, H., Yu, R., \& Li, X. 2016. A microbial fuel cell-coupled constructed wetland promotes degradation of azo dye decolorization products. Ecological Engineering. 94: 455-463.

Faulwetter, J. L., Gagnon, V., Sundberg, C., Chazarenc, F., Burr, M. D., Brisson, J., \& Stein, O. R. 2009. Microbial processes influencing performance of treatment wetlands: a review. Ecological Engineering. 35(6): 987-1004.

Gao, C., Liu, L., \& Yang, F. 2017. Development of a novel proton exchange membranefree integrated MFC system with electric membrane bioreactor and air contact oxidation bed for efficient and energy-saving wastewater treatment. Bioresource Technology. 238: $472-483$.

Gao, Y., Xie, Y. W., Zhang, Q., Wang, A. L., Yu, Y. X., \& Yang, L. Y. 2017. Intensified nitrate and phosphorus removal in an electrolysis-integrated horizontal subsurface-flow constructed wetland. Water Research: 108, 39-45. 
Ghangrekar, M. M., \& Shinde, V. B. 2007. Performance of membrane-less microbial fuel cell treating wastewater and effect of electrode distance and area on electricity production. Bioresource Technology. 98(15): 2879-2885.

Goldin, I., \& Winters, L. A. (Eds.). 1995. The economics of sustainable development. Cambridge University Press.

Guzmán, M. F. S., Ruiz, D. D. P., Galvis, F., Sierra, M. L. R., \& Torres, S. N. C. 2017. Análisis prospectivo del uso de energía solar: Caso Colombia. Investigación y Ciencia: de la Universidad Autónoma de Aguascalientes. (71): 85-93.

He, H., Zhou, M., Yang, J., Hu, Y., \& Zhao, Y. 2014. Simultaneous wastewater treatment, electricity generation and biomass production by an immobilized photosynthetic algal microbial fuel cell. Bioprocess and Biosystems Engineering. 37(5): 873-880.

Lefebvre, O., Ha Nguyen, T. T., Al-Mamun, A., Chang, I. S., \& Ng, H. Y. 2010. T-RFLP reveals high $\beta$-Proteobacteria diversity in microbial fuel cells enriched with domestic wastewater. Journal of Applied Microbiology. 109(3): 839-850.

Li, Z., Qian, C., Ding, Y., Bai, S., \& Lv, X. 2016. A review of microbial fuel cells coupled with constructed wetland. Advances in Engineering Research. (63):1-5

Liu, H., Cheng, S., \& Logan, B. E. 2005. Power generation in fed-batch microbial fuel cells as a function of ionic strength, temperature, and reactor configuration. Environmental Science \& Technology. 39(14): 5488-5493.

Liu, H., Cheng, S., Huang, L., \& Logan, B. E. 2008. Scale-up of membrane-free singlechamber microbial fuel cells. Journal of Power Sources. 179(1): 274-279.

Liu, R., Tursun, H., Hou, X., Odey, F., Li, Y., Wang, X., \& Xie, T. 2017. Microbial community dynamics in a pilot-scale MFC-AA/O system treating domestic sewage. Bioresource Technology. 241: 439-447.

Liu, S., Feng, X., \& Li, X. 2017. Bioelectrochemical approach for control of methane emission from wetlands. Bioresource Technology. 241: 812-820.

Liu, S., Song, H., Wei, S., Yang, F., \& Li, X. 2014. Bio-cathode materials evaluation and configuration optimization for power output of vertical subsurface flow constructed wetland-Microbial fuel cell systems. Bioresource Technology. 166: 575-583.

Liu, T., Chen, X., Wang, X., Zheng, S., \& Yang, L. 2018. Highly effective wastewater phosphorus removal by phosphorus accumulating organism combined with magnetic sorbent MFC@La (OH)3. Chemical Engineering Journal. 335: 443-449.

Logan, B. E. 2009. Exoelectrogenic bacteria that power microbial fuel cells. Nature Reviews Microbiology. 7(5): 375-381.

Logan, B. E., \& Elimelech, M. 2012. Membrane-based processes for sustainable power generation using water. Nature. 488(7411): 313-319. 
Logan, B. E., \& Rabaey, K. 2012. Conversion of wastes into bioelectricity and chemicals by using microbial electrochemical technologies. Science. 337(6095): 686-690.

Logan, B. E., Hamelers, B., Rozendal, R., Schröder, U., Keller, J., Freguia, S., \& Rabaey, K. 2006. Microbial fuel cells: methodology and technology. Environmental Science \& Technology. 40 (17): 5181-5192.

López, F. G. 2016. El proceso de coquización como vía de reciclado de residuos de polietileno (Doctoral, Universidad de Oviedo), Asturias España.

Lu, L., Xing, D., \& Ren, Z. J. 2015. Microbial community structure accompanied with electricity production in a constructed wetland plant microbial fuel cell. Bioresource Technology. 195: 115-121.

Mohan, S. V., Velvizhi, G., Modestra, J. A., \& Srikanth, S. 2014. Microbial fuel cell: critical factors regulating bio-catalyzed electrochemical process and recent advancements. Renewable and Sustainable Energy Reviews. 40: 779-797.

Nam, J. Y., Kim, H. W., Lim, K. H., \& Shin, H. S. 2010. Effects of organic loading rates on the continuous electricity generation from fermented wastewater using a single-chamber microbial fuel cell. Bioresource Technology. 101(1): S33-S37.

Oon, Y. L., Ong, S. A., Ho, L. N., Wong, Y. S., Dahalan, F. A., Oon, Y. S., \& Nordin, N. 2017. Role of macrophyte and effect of supplementary aeration in up-flow constructed wetland-microbial fuel cell for simultaneous wastewater treatment and energy recovery. Bioresource Technology. 224: 265-275.

Oon, Y. L., Ong, S. A., Ho, L. N., Wong, Y. S., Oon, Y. S., Lehl, H. K., \& Thung, W. E. 2015. Hybrid system up-flow constructed wetland integrated with microbial fuel cell for simultaneous wastewater treatment and electricity generation. Bioresource Technology. 186: $270-275$.

Partridge, T., Thomas, M., Harthorn, B. H., Pidgeon, N., Hasell, A., Stevenson, L., \& Enders, C. 2017. Seeing futures now: Emergent US and UK views on shale development, climate change and energy systems. Global Environmental Change. 42: 1-12.

Patil, S. A., Harnisch, F., Kapadnis, B., \& Schröder, U. 2010. Electroactive mixed culture biofilms in microbial bioelectrochemical systems: the role of temperature for biofilm formation and performance. Biosensors and Bioelectronics. 26(2): 803-808.

Rahimnejad, M., Adhami, A., Darvari, S., Zirepour, A., \& Oh, S. E. 2015. Microbial fuel cell as new technology for bioelectricity generation: a review. Alexandria Engineering Journal. 54(3): 745-756.

Roh, S. H., \& Woo, H. G. 2017. Microbial Fuel Cells. Kirk-Othmer Encyclopedia of Chemical Technology.

Salas, J. J., Aragón, C., Stich, M., Gonzalez, A., Machón, C., Esteve, R., \& Esteve-Núñez, A. 2017. METlands: A new generation of hybrid bioelectrochemical wetlands outperform 
standard wetlands for treating wastewater. In 7th International Symposium for Wetland Pollutant Dynamics and Control (WETPOL).

Saratale, G. D., Saratale, R. G., Shahid, M. K., Zhen, G., Kumar, G., Shin, H. S., \& Kim, S. H. 2017. A comprehensive overview on electro-active biofilms, role of exo-electrogens and their microbial niches in microbial fuel cells (MFCs). Chemosphere. 178: 534-547.

Sauer, K., Camper, A. K., Ehrlich, G. D., Costerton, J. W., \& Davies, D. G. 2002. Pseudomonas aeruginosa displays multiple phenotypes during development as a biofilm. Journal of Bacteriology. 184(4): 1140-1154.

Song, H., Zhang, S., Long, X., Yang, X., Li, H., \& Xiang, W. 2017. Optimization of bioelectricity generation in constructed wetland-coupled microbial fuel cell systems. Water. 9(3): 185.

Srivastava, P., Dwivedi, S., Kumar, N., Abbassi, R., Garaniya, V., \& Yadav, A. K. 2017. Performance assessment of aeration and radial oxygen loss assisted cathode based integrated constructed wetland-microbial fuel cell systems. Bioresource Technology. 244: 1178-1182.

Tee, P. F., Abdullah, M. O., Tan, I. A., Amin, M. A., Nolasco-Hipolito, C., \& Bujang, K. 2017. Effects of temperature on wastewater treatment in an affordable microbial fuel celladsorption hybrid system. Journal of Environmental Chemical Engineering. 5(1): 178-188.

Timmers, R. A., Strik, D. P., Hamelers, H. V., \& Buisman, C. J. 2010. Long-term performance of a plant microbial fuel cell with Spartina anglica. Applied Microbiology and Biotechnology. 86(3): 973-981.

Tsai, H. Y., Wu, C. C., Lee, C. Y., \& Shih, E. P. 2009. Microbial fuel cell performance of multiwall carbon nanotubes on carbon cloth as electrodes. Journal of Power Sources. 194(1): 199-205.

Türker, O. C., \& Yakar, A. 2017. A hybrid constructed wetland combined with microbial fuel cell for boron (B) removal and bioelectric production. Ecological Engineering. 102: 411-421.

Vessuri, H., \& de Montevideo, U. O. 2016. La ciencia para el desarrollo sostenible (Agenda 2030).

Villaseñor, J., Capilla, P., Rodrigo, M. A., Canizares, P., \& Fernández, F. J. 2013. Operation of a horizontal subsurface flow constructed wetland-microbial fuel cell treating wastewater under different organic loading rates. Water Research. 47(17): 6731-6738.

Wang, J., Song, X., Wang, Y., Abayneh, B., Ding, Y., Yan, D., \& Bai, J. 2016. Microbial community structure of different electrode materials in constructed wetland incorporating microbial fuel cell. Bioresource Technology. 221: 697-702.

Wang, J., Song, X., Wang, Y., Abayneh, B., Li, Y., Yan, D., \& Bai, J. 2016a. Nitrate removal and bioenergy production in constructed wetland coupled with microbial fuel cell: 
establishment of electrochemically active bacteria community on anode. Bioresource Technology. 221: 358-365.

Wang, J., Song, X., Wang, Y., Bai, J., Bai, H., Yan, D., \& Dong, G. 2017. Bioelectricity generation, contaminant removal and bacterial community distribution as affected by substrate material size and aquatic macrophyte in constructed wetland-microbial fuel cell. Bioresource Technology. 245: 372-378.

Wang, J., Song, X., Wang, Y., Bai, J., Li, M., Dong, G., \& Yan, D. 2017a. Bioenergy generation and rhizodegradation as affected by microbial community distribution in a coupled constructed wetland-microbial fuel cell system associated with three macrophytes. Science of the Total Environment. 607: 53-62.

Wen, Q., Wang, S., Yan, J., Cong, L., Pan, Z., Ren, Y., \& Fan, Z. 2012. $\mathrm{MnO}_{2}$-graphene hybrid as an alternative cathodic catalyst to platinum in microbial fuel cells. Journal of Power Sources. 216: 187-191.

Wu, S., Li, H., Zhou, X., Liang, P., Zhang, X., Jiang, Y., \& Huang, X. 2016. A novel pilotscale stacked microbial fuel cell for efficient electricity generation and wastewater treatment. Water Research. 98: 396-403.

Wu, S., Lv, T., Lu, Q., Ajmal, Z., \& Dong, R. 2017. Treatment of anaerobic digestate supernatant in microbial fuel cell coupled constructed wetlands: Evaluation of nitrogen removal, electricity generation, and bacterial community response. Science of the Total Environment. 580: 339-346.

Xu, D., Xiao, E. R., Xu, P., Zhou, Y., Zhou, Q. H., Xu, D., \& Wu, Z. B. 2017a. Effects of influent organic loading rates and electrode locations on the electrogenesis capacity of constructed wetland-microbial fuel cell systems. Environmental Progress and Sustainable Energy. 36(2): 435-441.

Xu, L., Zhao, Y., Doherty, L., Hu, Y., \& Hao, X. 2016. Promoting the bio-cathode formation of a constructed wetland-microbial fuel cell by using powder activated carbon modified alum sludge in anode chamber. Scientific Reports. 6.

Xu, L., Zhao, Y., Fan, C., Fan, Z., \& Zhao, F. 2017. First study to explore the feasibility of applying microbial fuel cells into constructed wetlands for COD monitoring. Bioresource Technology, 243, 846-854.

Yadav, A. K., Dash, P., Mohanty, A., Abbassi, R., \& Mishra, B. K. 2012. Performance assessment of innovative constructed wetland-microbial fuel cell for electricity production and dye removal. Ecological Engineering. 47: 126-131.

Yang, Q., Wu, Z., Liu, L., Zhang, F., \& Liang, S. 2016. Treatment of oil wastewater and electricity generation by integrating constructed wetland with microbial fuel cell. Materials. 9(11): 885 .

Zhang, G., Jiao, Y., \& Lee, D. J. 2015. A lab-scale anoxic/oxic-bioelectrochemical reactor for leachate treatments. Bioresource Technology. 186: 97-105. 
Zhang, S., Yang, X. L., Li, H., Song, H. L., Wang, R. C., \& Dai, Z. Q. 2017. Degradation of sulfamethoxazole in bioelectrochemical system with power supplied by constructed wetland-coupled microbial fuel cells. Bioresource Technology. 244: 345-352.

Zhang, X., Ye, X., Finneran, K. T., Zilles, J. L., \& Morgenroth, E. 2013. Interactions between Clostridium beijerinckii and Geobacter metallireducens in co-culture fermentation with anthrahydroquinone-2, 6-disulfonate (AH2QDS) for enhanced biohydrogen production from xylose. Biotechnology and Bioengineering. 110(1): 164-172.

Zhao, Y., Collum, S., Phelan, M., Goodbody, T., Doherty, L., \& Hu, Y. 2013. Preliminary investigation of constructed wetland incorporating microbial fuel cell: batch and continuous flow trials. Chemical Engineering Journal. 229: 364-370.

Zhi, W., Yuan, L., Ji, G., \& He, C. 2015. Enhanced long-term nitrogen removal and its quantitative molecular mechanism in tidal flow constructed wetlands. Environmental Science \& Technology. 49(7): 4575-4583.

Zhou, M., Wang, H., Hassett, D. J., \& Gu, T. 2013. Recent advances in microbial fuel cells (MFCs) and microbial electrolysis cells (MECs) for wastewater treatment, bioenergy and bioproducts. Journal of Chemical Technology and Biotechnology. 88(4): 508-518. 